\title{
Tagungsbericht
}

\author{
Tamara Heck*
}

\section{Open Access und Open Science in der Hochschullehre}

\section{DOI 10.1515/iwp-2016-0065}

„Ich als Student wünsche mir kostenlosen Zugriff auf so viele wissenschaftliche Dokumente wie möglich und kann daher von Open Access sehr profitieren.“ Diese Meinung vertritt Philipp Nowak, Student der Informationswissenschaft an der Heinrich-Heine-Universität Düsseldorf. Er ist für Open Access und kann nicht verstehen, warum nicht alle Literatur gratis für ihn zugänglich ist. Sein Kommilitone Jan Scheddler differenziert seine Aussage: „Als Verlagschef bin ich der Meinung, dass Open Access in das Portfolio eines jeden guten Wissenschaftsverlages gehört. Die Vorstellung, dass es für alle Beteiligten kostenlos ist, ist allerdings zu idealistisch.“

Die strikten Positionen der beiden Studierenden sind hier fiktiv, beide haben eine bestimmte Rolle eingenom- men, um in der abschließenden Podiumsdiskussion verschiedene Standpunkte zum Thema Open Access und Open Science zu vertreten. Nicht jeder vertritt seine eigene Meinung, aber durch die verschiedenen Ansichten wird den Gästen verdeutlicht, dass die Debatte um den Umgang und die Entwicklung von Open Access nicht immer so einfach ist, wie sie zu sein scheint. Wissenschaftler und Studierende diskutieren hier mit Verlagen und Bibliotheken. Am Ende ziehen die Teilnehmer das Fazit, dass sich viele über das grundsätzlich Positive zu Open Access und Open Science einig sind, es aber bei der Umsetzung noch eine ganze Reihe an Probleme zu geben scheint.

Die Studierenden der Informationswissenschaft in Düsseldorf diskutierten nicht in einem geschlossenen Seminar, sondern vor Gästen bei ihrer selbst organisierten

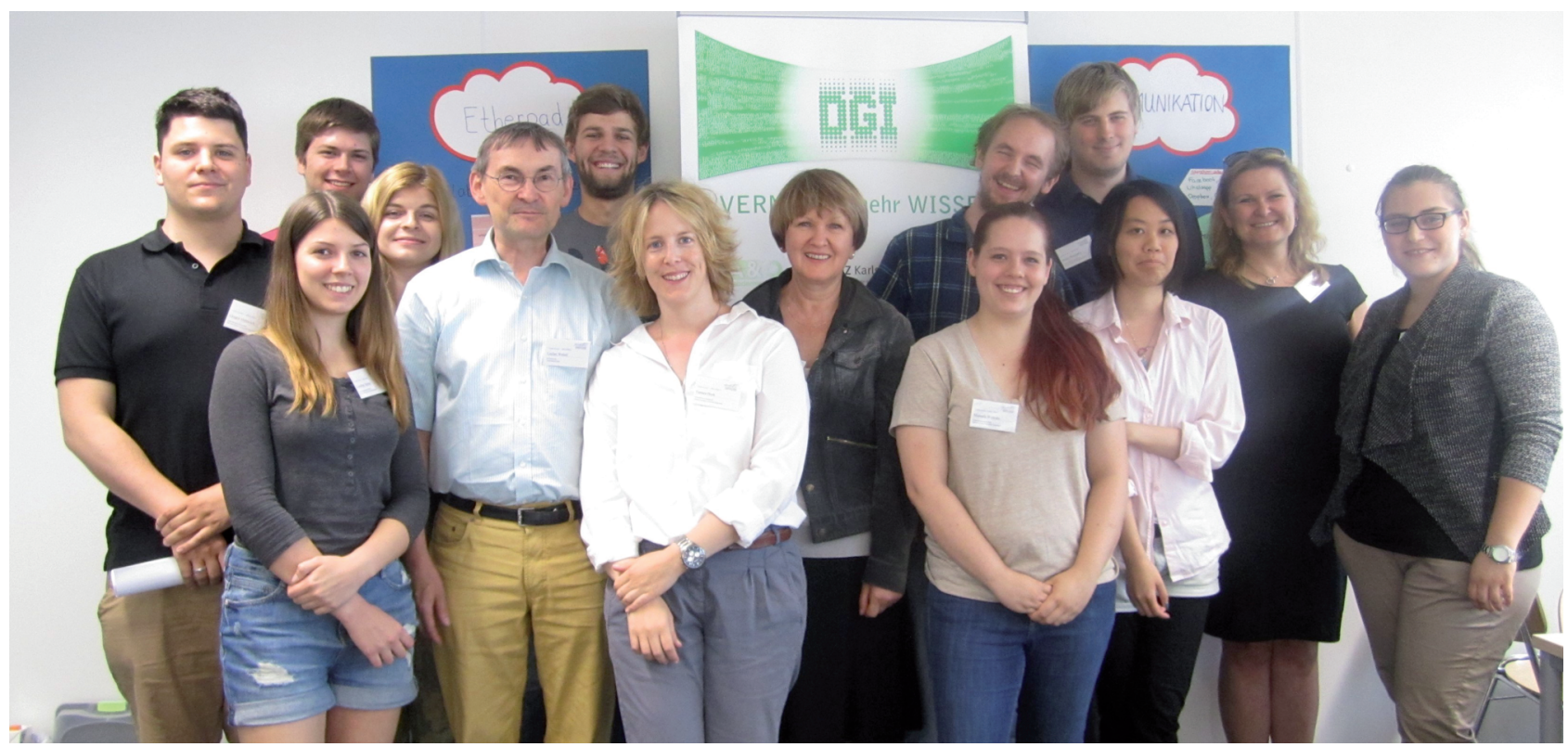

Abbildung 1: Seminarorganisatoren und Gäste (Foto: Marion Herstell).

*Kontaktperson: Dr. Tamara Heck, Institut für Sprache und Information, Abteilung für Informationswissenschaft, Heinrich-Heine-Universität Düsseldorf, Universitätsstraße 1, 40225 Düsseldorf, E-Mail: tamara.heck@hhu.de 
Konferenz „</open.access $>$ - alles offen?!“, die im Juli 2016 in Düsseldorf stattfand. Zwölf Studierende beschäftigten sich im Rahmen eines Kurses unter diversen Gesichtspunkten mit der Open Access-Bewegung und entwarfen ein kleines Programm mit denen für sie relevanten Aspekten des Themas. Mit Unterstützung der Deutschen Gesellschaft für Information und Wissen e. V. (DGI) waren die Studierenden in der Lage, eine kleine Konferenz $\mathrm{zu}$ organisieren, bei der alle Interessierten teilnehmen konnten - gemäß dem Thema „alles offen!“. DGI Schatzmeisterin Sigrid Riedel (Vorstand WTI-Frankfurt) und Vizepräsident Dr. Luzian Weisel (FIZ Karlsruhe) waren als Gäste anwesend. Sie berichteten von ihren Erfahrungen mit Open Access und auch von Kollaborationstools in den Unternehmen. Rege Diskussionen mit den Studierenden gab es unter anderem bei der Frage „Wie komme ich an meine Literatur?" Denn neben dem grünen und goldenen Weg kannten die Studierenden auch den dunkelgrauen und den schwarzen Weg. Diese rechtlich fragwürdigen Möglichkeiten würden genutzt, so argumentierten einige der Studierenden, weil einfache und schnelle Alternativen fehlten, die zum Beispiel von Bibliotheken angeboten werden könnten.

Insgesamt gab es sechs Vorträge und eine Podiumsdiskussion, bei der fünf Teilnehmer unterschiedliche Positionen vertraten und versuchten, sich beispielsweise in die Lage von Bibliotheken und Verlagen hineinzuversetzen. Neben einer Einführung zur Geschichte von Open Access beschäftigen sich die Vorträge mit weiteren Aspekten: So stellte Blerta Kamberi die Institutionen, die sich für Open Access einsetzen, sowie deren Initiativen und Programme zur Förderung der Bewegung vor. Ein weiteres Thema waren offene Forschungsdaten, das die Teilnehmerin Emilia Palka-Binkiewicz näher erläuterte. Aber auch das kritische Portal SciHub wurde sehr kontrovers von den Gästen und dem Referenten Gero Wollgarten diskutiert. Viele Studie- rende interessierten besonders Themen, zu denen sie im Studium und Alltag einen direkten Bezug haben, wie Massive Open Online Courses sowie Austausch- und Kollaborationstools für Studierende, die das offenen Lernen fördern sollen. Diese wurden beispielsweise durch ein Wiki und digitale Pads im Kurs angewendet und getestet. Vor allem die einfachen Editorenpads für Protokolle und die Konferenzplanung erwiesen sich als sehr nützlich.

Aktuell wird das Thema Open Science in der Hochschullehre auch im Leibniz-Forschungsverbund Science 2.0 (https://www.leibniz-science20.de/de/) erörtert. Diskussionen gab es in der Forschergruppe unter anderem auf dem Open Educational Resources Festival sowie der Science 2.0-Konferenz. Ziel ist es, das Thema in die Hochschulen zu tragen sowie Dozierende für das Thema zu sensibilisieren, weil diese in ihren Einrichtungen zukünftige Wissenschaftler ausbilden.

Link zur Konferenzwebseite: http://openaccess.phil.hhu. de/

DGI-Blog: http://dgi-info.de/erfahrung-trifft-neugier-rueckblick-auf-die-studentische-konferenz-open-access-alles-of fen-in-duesseldorf/

Deskriptoren: Tagung, Open Access, Hochschulausbildung, Forschung, Universität Düsseldorf

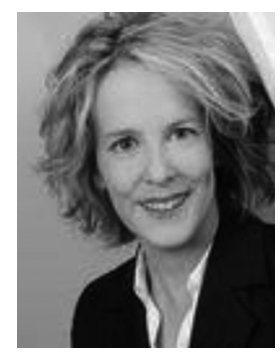

Dr. Tamara Heck Institut für Sprache und Information Abteilung für Informationswissenschaft Heinrich-Heine-Universität Düsseldorf Universitätsstraße 1 40225 Düsseldorf tamara.heck@hhu.de 